\title{
Malignant Cylindroma- A Rare Case Diagnosis Made on Cytology
}

\author{
Dr. Navneet Kaur ${ }^{1}$, Dr. Kanwardeep Kaur ${ }^{2}$, Dr. Shaffaly Gagneja ${ }^{3 *}$, Dr. Haspreet Kaur Gill ${ }^{4}$
}

\author{
${ }^{1}$ Associate Professor, Department of Pathology, GMC, Patiala, Sangrur Road, Punjab 147001, India \\ ${ }^{2}$ Assistant Professor, Department of Pathology, GMC, Patiala, Sangrur Road, Punjab 147001, India \\ ${ }^{3}$ Junior resident, Department of Pathology, GMC, Patiala, Sangrur Road, Punjab 147001, India \\ ${ }^{4}$ Junior resident, Department of Pathology, GMC, Patiala, Sangrur Road, Punjab 147001, India
}

DOI: $10.36347 /$ sjmcr.2021.v09i03.004

| Received: 18.02.2021 | Accepted: 01.03.2021 | Published: 06.03.2021

*Corresponding author: Shaffaly Gagneja

Abstract

Case Report

Malignant cylindroma (cylindrocarcinoma) is an extremely rare adnexal neoplasm. Malignant cylindroma may develop via malignant transformation of long standing benign cylindroma or may develop denovo in others [1]. There are few cases of malignant transformation of dermal cylindromas in the literature [2]. Here we report a rare case of malignant cylindroma reported on cytology in a 56 year old male patient with a solitary lesion on occipital region.

Keywords: Malignant cylindroma, adnexal neoplasm, cytology.

Copyright $(\odot 2021$ The Author(s): This is an open-access article distributed under the terms of the Creative Commons Attribution 4.0 International License (CC BY-NC 4.0) which permits unrestricted use, distribution, and reproduction in any medium for non-commercial use provided the original author and source are credited.

\section{INTRODUCTION}

Cylindromas are rare tumours of the apocrine or eccrine glands and are classified in the family of skin adnexal tumors. The term cylindroma was first used by Billroth in 1859 to describe an orbital tumour with cylindrical shapes [3]. Benign cylindromas (BC) occur as solitary or multiple lesions. The lesions are usually slow-growing tumors. The presence of multiple cylindromas is associated with hereditary conditions including familial cylindromatosis (FC) and BrookeSpiegler syndrome (BSS). These familial syndromes have been shown to have an increased incidence for malignant transformation of benign lesions. In rare instances, malignant cylindromas (MC) occur in patients without any known hereditary risks. They tend to behave aggressively with a high risk of recurrence and metastasis. Malignant Cylindroma was first described by Wiedmann in 1929 on the scalp of 63 year old woman. Less than 50 cases of MC have been reported in the literature [4]. Clinically, cylindrocarcinomas most commonly are localized on the head and neck [1]. FNAC is a great tool for diagnosing malignant cylindroma as it is easy to perform and an inexpensive method.

\section{Case Report}

A 56 year old male patient presented in the department of pathology with multiple swellings in cervical region. On taking further history, patient revealed another swelling on occipital region which was present since 25 years. There was sudden increase in the size of occipital swelling since 15 days. No radiological investigation was done.

FNA was done using 22 Gauge needle attached to $10 \mathrm{ml}$ syringe on camecohandle under aseptic precautions from both the sites. Smears were air dried and stained with MGG.

Cytological findings were similar from both aspirations. Smears were highly cellular showing bimodal population of malignant epithelial cells in the background of some stromal fragments and hyalinized pink material. The scattered population of cells were large exhibiting moderate nuclear atypia and prominent macronucleoli in many with variable granular cytoplasm. Binucleate cells and bare nuclei were also seen. Other population comprised of small to medium sized cells having hyperchromatic nuclei and moderate amount of cytoplasm. 
Based on these findings and correlating with the site a diagnosis of malignant adnexal tumour favouring malignant cylindroma was made provisionally.

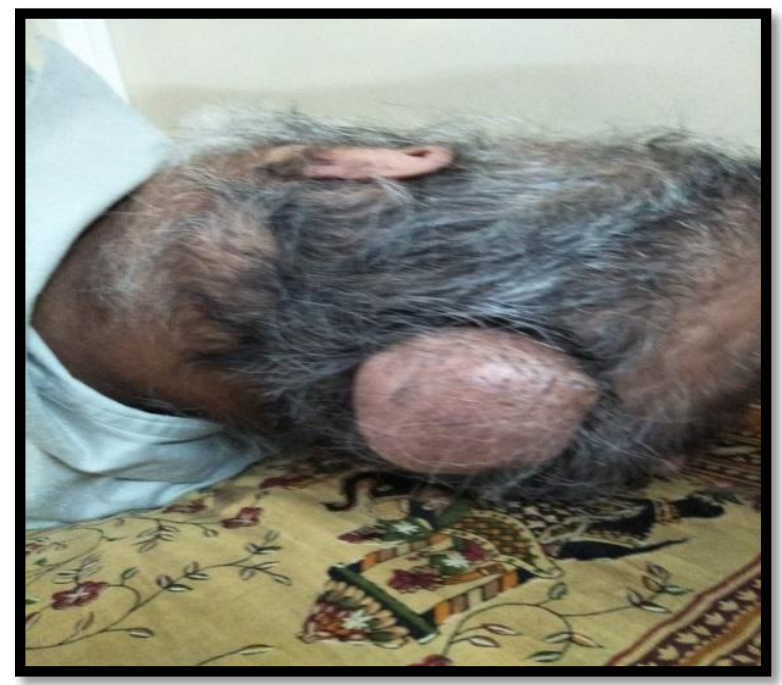

Fig-1: Gross picture of occipital swelling

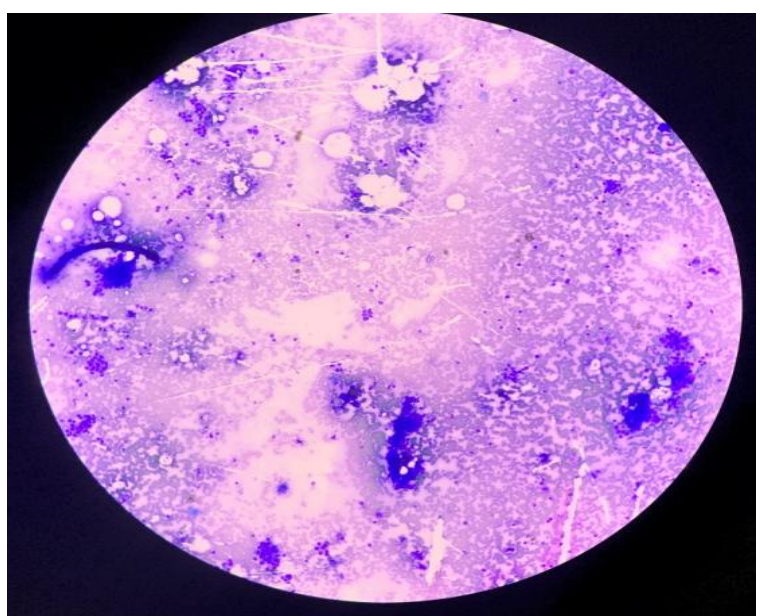

Fig-2: Cellular smear showing malignant epithelial cells

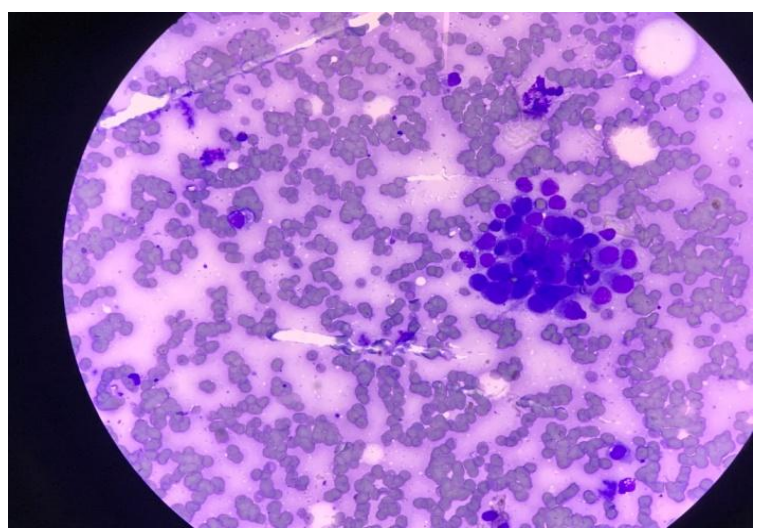

Fig-3: Smear showing bimodal population of malignant epithelial cells

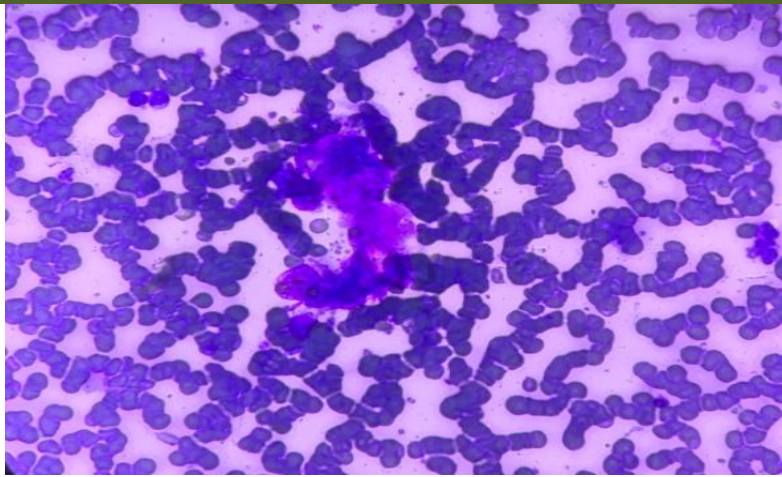

Fig-4: Smear showing hyalinised pink material

\section{DISCUSSION}

Cutaneous cylindromas are pink skin coloured growth. It mainly occurs on head and neck region. It can either be solitary or multiple. Solitary cylindroma occur sporadically and are not inherited. Multiple lesions may cover entire scalp giving the appearance of disfiguring turban, hence the name turban tumour. Ratio of females to males is 2:1. Lesion appear in second decade of life and growth is gradual.The lesions are usually painless but $35 \%$ patients reported the same to be painful [5].

Malignant transformation of cutaneous cylindroma is very rare and is usually seen in multiple type or in long standing benign neoplasm. Malignant transformation is characterised by rapid growth, ulceration, bleeding, colour change, pain and fixation of lesion.

In our patient, there was a long history of swelling since 25 years in occipital region. The swelling had a surface ulceration associated with pus like discharge.Malignant cylindroma is usually locally aggressive tumour.It spreads along draining lymphatic vessels [5]. Using pubmed database research, we found that total of 36 well documented cases of malignant cylindroma in the literature. Out of these 36 cases,only 9 transformed from solitary type [6].

Immunohistochemically, cylindroma express markers indicating derivation from both eccrine and apocrine glands. IHC expression of S 100, Alpha smooth muscle actin, EMA, CEA, laminin, collagen $1 \mathrm{~V}$, fibronectin and CD 34 are seen. IHC is not helpful in distinguishing benign from malignant cylindroma [5]. Cell block was not prepared in the present case. So, IHC was performed showing positivity for S 100 .

The preferred mode of treatment for solitary lesions is wide local excision as it has high recurrence rate. Other treatment modalities are moh'smicrographic surgery, carbon dioxide laser and radiotherapy. 
Navneet kaur et al., Sch J Med Case Rep, Mar, 2021; 9(3): 211-213

Mulitple cylindromas require extensive plastic surgery along with excising a group of nodules in multiple settings. Once transformation takes place, the tendency for local destruction as well as metastasis rates get increased. To ensure a better prognosis early diagnosis and close follow up is mandatory in patient with multiple cylindromas [6].

\section{REFERNCES}

1. Borik L, Heller P, Shrivastava M, Kazlouskaya V. Malignant cylindroma in a patient with BrookeSpiegler syndrome. Dermatol Pract Concept. 2015;5(2):61- 65.

2. Akgul GG, Yenidogan E, Dinc S, Pak I, Colakoglu MK, Gulcelik MA. Malignant cylindroma of the scalp with multiple cervical lymph node metastasis-A case report. Int J Surg Case Rep. 2013;4(7):589- 592.

3. Tosun Z, Hosnuter M, Senturk S, Avunduk MC, Savaci N. Cylindroma of the foot. Ann Plast Surg. 2002;48:220-221.

4. Kim SM, Truong JL, Wu K, Gabril MY, Grant A. Malignant cylindroma of the scalp treated with staged perimeter excision: A case report and literature review. JPRAS Open. 2019;21:1- 5.

5. Bansal C, Batra M, Lal N, Srivastava AN. Solitary cylindroma with malignant transformation. Indian J Dermatol. 2012;57(2):141- 143. doi:10.4103/0019-5154.94289

6. Kuklani RM, Glavin FL, Bhattacharyya I. Malignant cylindroma of the scalp arising in a setting of multiple cylindromatosis: a case report. Head Neck Pathol. 2009;3(4):315- 319. 